\title{
Fractional Statistical Mechanics
}

\author{
Vasily E. Tarasov \\ Skobeltsyn Institute of Nuclear Physics, \\ Moscow State University, Moscow 119991, Russia \\ E-mail: tarasov@theory.sinp.msu.ru
}

\begin{abstract}
The Liouville and first Bogoliubov hierarchy equations with derivatives of noninteger order are derived. The fractional Liouville equation is obtained from the conservation of probability to find a system in a fractional volume element. This equation is used to obtain Bogoliubov hierarchy and fractional kinetic equations with fractional derivatives. Statistical mechanics of fractional generalization of the Hamiltonian systems is discussed. Liouville and Bogoliubov equations with fractional coordinate and momenta derivatives are considered as a basis to derive fractional kinetic equations. The Fokker-Planck-Zaslavsky equation that has fractional phase-space derivatives is obtained from fractional Bogoliubov equation. The linear fractional kinetic equation for distribution of the charged particles is considered.
\end{abstract}

The theory of integrals and derivatives of noninteger order goes back to Leibniz, Liouville, Riemann, Grunwald, and Letnikov. Fractional calculus has found many applications in recent studies in mechanics and physics. Fractional equations, which have derivatives of noninteger order, are very successful in describing anomalous kinetics, transport, and chaos. Fractional kinetics equations usually appear from some phenomenological models. In this paper, we suggest fractional equations of statistical mechanics. To obtain these equations, the conservation of probability to find a system in a fractional differential volume element of the phase-space is used. This element can be considered as a small part of the phase-space set with noninteger dimension. The suggested fractional equations of statistical mechanics are used to derive the fractional kinetics equations. 


\section{Introduction}

Fractional equations [1, 2] contain derivatives of noninteger order [3, 4]. Integrals and derivatives of fractional order have found many applications in recent studies in mechanics and physics. In a short period of time the list of such applications becomes long. For example, it includes chaotic dynamics [5, 6], mechanics of fractal media [7, 8, 9], quantum mechanics [10, 11], physical kinetics [5, 12, 13, 14, 15], plasmas physics [16, 17], long-range dissipation [18, 19, 20, mechanics of non-Hamiltonian systems [21, 22, theory of long-range interaction [23, 24, 25], anomalous diffusion, and transport theory [5, 26, 27].

Equations, which involve derivatives or integrals of noninteger order are very successful in describing anomalous kinetics [5, 6, 12, 13, 14]. Usually the fractional equations in dynamics or kinetics appear as some phenomenological models. In [21] the attempt to derive the basic statistical mechanics equations with derivatives of noninteger order have been realized. Unfortunately, the fractional derivatives appear only by Fourier transform of these equations as it realized for the Fokker-Planck-Zaslavsky equation in [9].

In this paper, we derive the Liouville equation with fractional derivatives with respect to coordinates and momenta. To obtain the fractional Liouville equation (FLE), we consider the conservation of probability in the fractional differential volume element. This element can be considered as a small part of the phase-space set with noninteger dimension. Using the FLE, we get a fractional generalization of the Bogoliubov hierarchy equations. These equations can be used to derive fractional kinetic equations [5, 6, 12, 13. The Vlasov equation with derivatives of noninteger order is obtained. The Fokker-Planck-Zaslavsky equation, which has fractional phase-space derivatives, is derived from the FLE. The linear fractional kinetic equation for distribution of the charged particles is suggested.

In Sec. 2, we obtain the Liouville equation with fractional derivatives from the conservation of probability in the fractional volume element of phase space. In Sec. 3, the first Bogoliubov hierarchy equation with fractional derivatives in phase space is derived. In Sec. 4, we consider the Vlasov equation with fractional derivatives in phase space. In Sec. 5, the Fokker-Planck-Zaslavsky equation, which has fractional derivatives with respect to coordinates and momenta is considered. In Sec. 6, the linear fractional kinetic equation for distribution of the charged particles is obtained. Finally, a short conclusion is given in Sec. 7 . 


\section{Liouville equation with fractional derivatives}

A basic principle of statistical mechanics is the conservation of probability in the phasespace. The Liouville equation is an expression of the principle in a convenient form for the analysis. In this section, we derive the Liouville equation with fractional derivatives from the conservation of probability in a fractional volume element.

In the phase space $R^{2 n}$ with coordinates $\left(x^{1}, \ldots, x^{2 n}\right)=\left(q_{1}, \ldots, q_{n}, p_{1}, \ldots, p_{n}\right)$, we consider a fractional differential volume element

$$
d^{\alpha} V=d^{\alpha} x_{1} \ldots d^{\alpha} x_{2 n}
$$

Here, $d^{\alpha}$ is a fractional differential [28] that is defined by

$$
d^{\alpha} f(x)=\sum_{k=1}^{2 n} D_{x_{k}}^{\alpha} f(x)\left(d x_{k}\right)^{\alpha}
$$

where $D_{x_{k}}^{\alpha}$ is a fractional derivative [3] of order $\alpha$ with respect to $x_{k}$.

The fractional derivative has different definitions [4, 3], and exploiting any of them depends on the initial (boundary) conditions, and the specifics of the considered physical processes. The classical definition is the so-called Riemann-Liouville derivative [3]. Due to reasons, concerning the initial and boundary conditions, it is more convenient to use the Caputo fractional derivatives [29, 1]. Its main advantage is that the initial conditions take the same form as for integer-order differential equations. The Caputo derivative is defined by

$$
D_{x}^{\alpha} f(x)={ }_{0}^{C} D_{x}^{\alpha} f(x)=\frac{1}{\Gamma(n-\alpha)} \int_{0}^{x} \frac{f^{(n)}(z)}{(x-z)^{\alpha+1-n}} d z,
$$

where $n-1<\alpha<n$, and $f^{(n)}(z)=d^{n} f(z) / d z^{n}$. Note that $D_{x_{k}}^{\alpha} 1=0$, and $D_{x_{k}} x_{l}=0$, where $(k \neq l)$. Using (2), we obtain

$$
d^{\alpha} x_{k}=D_{x_{k}}^{\alpha} x_{k}\left(d x_{k}\right)^{\alpha}
$$

Then

$$
\left(d x_{k}\right)^{\alpha}=\left(D_{x_{k}}^{\alpha} x_{k}\right)^{-1} d^{\alpha} x_{k}
$$

From (3),

$$
D_{x_{k}}^{\alpha} x_{k}^{\beta}=\frac{\Gamma(\beta+1)}{\Gamma(\beta+1-\alpha)} x_{k}^{\beta-\alpha},
$$

where $\beta>\alpha>0$. Equations (44) and (6) give

$$
\left(d x_{k}\right)^{\alpha}=\Gamma(2-\alpha) x_{k}^{\alpha-1} d^{\alpha} x_{k} .
$$


The conservation of probability for the usual phase-space volume element is expressed as

$$
-d V \frac{\partial \rho(t, x)}{\partial t}=d[\rho(t, x)(\mathbf{u}, d \mathbf{S})]
$$

For the fractional volume element (11),

$$
-d^{\alpha} V \frac{\partial \rho(t, x)}{\partial t}=d^{\alpha}\left[\rho(t, x)\left(\mathbf{u}, d^{\alpha} \mathbf{S}\right)\right]
$$

Here, $\rho=\rho(t, x)$ is the density of probability to find the dynamical system in $d^{\alpha} V, \mathbf{u}=\mathbf{u}(t, x)$ is the velocity vector field in $R^{2 n}, d^{\alpha} \mathbf{S}$ is a surface element, and the brackets (, ) is a scalar product of vectors

$$
\mathbf{u}=\sum_{k=1}^{2 n} u_{k} \mathbf{e}_{k}, \quad d^{\alpha} \mathbf{S}=\sum_{k=1}^{2 n} d^{\alpha} S_{k} \mathbf{e}_{k}, \quad\left(\mathbf{u}, d^{\alpha} \mathbf{S}\right)=\sum_{k=1}^{2 n} u_{k} d^{\alpha} S_{k}
$$

where $\mathbf{e}_{k}$ are the basic vectors of Cartesian coordinate system, and

$$
d^{\alpha} S_{k}=d^{\alpha} x_{1} \ldots d^{\alpha} x_{k-1} d^{\alpha} x_{k+1} \ldots d^{\alpha} x_{2 n}
$$

The functions $u_{k}=u_{k}(t, x)$ define $x_{k}$ components of $\mathbf{u}(t, x)$. In the usual case $(\alpha=1)$, the outflow of the probability in the $x_{k}$ direction is

$$
d\left[\rho u_{k}\right] d S_{k}=D_{x_{k}}\left[\rho u_{k}\right] d x_{k} d S_{k}=D_{x_{k}}\left[\rho u_{k}\right] d V
$$

For $\alpha \neq 1$,

$$
d^{\alpha}\left[\rho u_{k}\right] d^{\alpha} S_{k}=D_{x_{k}}^{\alpha}\left[\rho u_{k}\right](d x)^{\alpha} d^{\alpha} S_{k} .
$$

Using (11), (1) and (51), we get

$$
d^{\alpha}\left[\rho u_{k}\right] d^{\alpha} S_{k}=D_{x_{k}}^{\alpha}\left[\rho u_{k}\right]\left(D_{x_{k}}^{\alpha} x_{k}\right)^{-1} d^{\alpha} x_{k} d^{\alpha} S_{k}=\left(D_{x_{k}}^{\alpha} x_{k}\right)^{-1} D_{x_{k}}^{\alpha}\left[\rho u_{k}\right] d^{\alpha} V .
$$

Substitution of (13) into (9) gives

$$
-d^{\alpha} V \frac{\partial \rho}{\partial t}=d^{\alpha} V \sum_{k=1}^{2 n}\left(D_{x_{k}}^{\alpha} x_{k}\right)^{-1} D_{x_{k}}^{\alpha}\left[\rho u_{k}\right] .
$$

As a result, we obtain

$$
\frac{\partial \rho}{\partial t}=-\sum_{k=1}^{2 n} \mathbf{D}_{x_{k}}^{\alpha}\left(\rho u_{k}\right)
$$

where

$$
\mathbf{D}_{x_{k}}^{\alpha}=\left(D_{x_{k}}^{\alpha} x_{k}\right)^{-1} D_{x_{k}}^{\alpha}=\Gamma(2-\alpha) x_{k}^{\alpha-1} D_{x_{k}}^{\alpha}
$$


This is the Liouville equation with the derivatives of fractional order $\alpha$. Equation (15) describes the probability conservation for the fractional volume element (1) of the phase space.

For the coordinates $\left(q_{1}, \ldots, q_{n}, p_{1}, \ldots, p_{n}\right)$, Eq. (15) is

$$
\frac{\partial \rho}{\partial t}+\sum_{k=1}^{n} \mathbf{D}_{q_{k}}^{\alpha}\left(\rho V_{k}\right)+\sum_{k=1}^{n} \mathbf{D}_{p_{k}}^{\alpha}\left(\rho F_{k}\right)=0,
$$

where $V_{k}=u_{k}$, and $F_{k}=u_{k+n}(k=1, \ldots, n)$. The functions $V_{k}=V_{k}(t, q, p)$ are the components of velocity field, and $F_{k}=F_{k}(t, q, p)$ are the components of the force field.

In general,

$$
D_{p_{k}}^{\alpha}\left[\rho F_{k}\right] \neq \rho D_{p_{k}}^{\alpha} F_{k}+F_{k} D_{p_{k}}^{\alpha} \rho .
$$

If $F_{k}$ does not depend on $p_{k}$, and $V_{k}$ does not depend on $q_{k}$, then Eq. (17) gives

$$
\frac{\partial \rho}{\partial t}+\sum_{k=1}^{n}\left(V_{k} \mathbf{D}_{q_{k}}^{\alpha} \rho+F_{k} \mathbf{D}_{p_{k}}^{\alpha} \rho\right)=0 .
$$

For the fractional generalization of Hamiltonian system [22], $V_{k}$ and $F_{k}$ can be presented as

$$
V_{k}=\mathbf{D}_{p_{k}}^{\alpha} H(q, p), \quad F_{k}=-\mathbf{D}_{q_{k}}^{\alpha} H(q, p),
$$

where $H(q, p)$ is a fractional generalization of Hamiltonian. Substitution of (20) into (19) leads to

$$
\frac{\partial \rho}{\partial t}+\sum_{k=1}^{n}\left(\mathbf{D}_{p_{k}}^{\alpha} H \mathbf{D}_{q_{k}}^{\alpha} \rho-\mathbf{D}_{q_{k}}^{\alpha} H \mathbf{D}_{p_{k}}^{\alpha} \rho\right)=0 .
$$

We can define

$$
\begin{gathered}
\{A, B\}_{\alpha}=\sum_{k=1}^{n}\left(\mathbf{D}_{q_{k}}^{\alpha} A \mathbf{D}_{p_{k}}^{\alpha} B-\mathbf{D}_{q_{k}}^{\alpha} B \mathbf{D}_{p_{k}}^{\alpha} A\right)= \\
=\sum_{k=1}^{n}\left(D_{q_{k}}^{\alpha} q_{k} D_{p_{k}}^{\alpha} p_{k}\right)^{-1}\left(D_{q_{k}}^{\alpha} A D_{p_{k}}^{\alpha} B-D_{q_{k}}^{\alpha} B D_{p_{k}}^{\alpha} A\right) .
\end{gathered}
$$

For $\alpha=1$, Eq. (221) gives the Poisson brackets. Note that

$$
\{A, B\}_{\alpha}=-\{B, A\}_{\alpha}, \quad\{1, A\}_{\alpha}=0 .
$$

Using (22), we get (21) in the form

$$
\frac{\partial \rho}{\partial t}+\{\rho, H\}_{\alpha}=0 .
$$

As the result, we have the Liouville equation for fractional generalization of Hamiltonian systems [22]. For $\alpha=1$, Eq. (23) is the usual Liouville equation. 


\section{Bogoliubov equation with fractional derivatives}

Let us consider a classical system with fixed number $N$ of identical particles. Suppose that $k$ th particle is described by the generalized coordinates $q_{k s}$ and generalized momenta $p_{k s}$, where $s=1, \ldots, m$. We use the notations $\mathbf{q}_{k}=\left(q_{k 1}, \ldots, q_{k m}\right)$ and $\mathbf{p}_{k}=\left(p_{k 1}, \ldots, p_{k m}\right)$. The state of this system can be described by the distribution function $\rho_{N}$ in the $2 m N$-dimensional phase space:

$$
\rho_{N}(\mathbf{q}, \mathbf{p}, t)=\rho\left(\mathbf{q}_{1}, \mathbf{p}_{1}, \ldots, \mathbf{q}_{N}, \mathbf{p}_{N}, t\right) .
$$

The normalization condition is

$$
\hat{I}[1, \ldots, N] \rho_{N}(\mathbf{q}, \mathbf{p}, t)=1
$$

where $\hat{I}[1, \ldots, N]$ is the integration with respect to $\mathbf{q}_{1}, \mathbf{p}_{1}, \ldots, \mathbf{q}_{N}, \mathbf{p}_{N}$.

The fractional Liouville equation is

$$
\frac{\partial \rho_{N}}{\partial t}=-\sum_{k=1}^{N}\left(\mathbf{D}_{\mathbf{q}_{\mathbf{k}}}^{\alpha}\left(\mathbf{V}_{k} \rho_{N}\right)+\mathbf{D}_{\mathbf{p}_{\mathbf{k}}}^{\alpha}\left(\mathbf{F}_{k} \rho_{N}\right)\right),
$$

where $\mathbf{V}_{k}$ is a velocity of $k$ th particle, $\mathbf{F}_{k}$ is the force that acts on $k$ th particle, and

$$
\begin{aligned}
& \mathbf{D}_{\mathbf{q}_{\mathbf{k}}}^{\alpha} \mathbf{V}_{k}=\left(D_{\mathbf{q}_{k}}^{\alpha} \mathbf{q}_{k}\right)^{-1} D_{\mathbf{q}_{\mathbf{k}}}^{\alpha} \mathbf{V}_{k}=\sum_{s=1}^{m}\left(D_{q_{k s}}^{\alpha} q_{k s}\right)^{-1} D_{q_{k s}}^{\alpha} V_{k s}, \\
& \mathbf{D}_{\mathbf{p}_{\mathbf{k}}}^{\alpha} \mathbf{F}_{k}=\left(D_{\mathbf{p}_{k}}^{\alpha} \mathbf{p}_{k}\right)^{-1} D_{\mathbf{p}_{\mathbf{k}}}^{\alpha} \mathbf{F}_{k}=\sum_{s=1}^{m}\left(D_{q_{k s}}^{\alpha} p_{k s}\right)^{-1} D_{p_{k s}}^{\alpha} F_{k s} .
\end{aligned}
$$

The one-particle reduced distribution function $\rho_{1}$ can be defined by

$$
\rho_{1}(\mathbf{q}, \mathbf{p}, t)=\rho\left(\mathbf{q}_{1}, \mathbf{p}_{1}, t\right)=\hat{I}[2, \ldots, N] \rho_{N}(\mathbf{q}, \mathbf{p}, t)
$$

where $\hat{I}[2, \ldots, N]$ is an integration with respect to $\mathbf{q}_{2}, \ldots, \mathbf{q}_{N}, \mathbf{p}_{2}, \ldots, \mathbf{p}_{N}$. Obviously, that the function (27) satisfies the normalization condition $\hat{I}[1] \rho_{1}(\mathbf{q}, \mathbf{p}, t)=1$.

The Bogoliubov hierarchy equations [30, 31, 32, 33] describe the evolution of the reduced distribution functions, and can be derived from the Liouville equation. To obtain the first Bogoliubov equation with fractional derivatives from Eq. (24) we consider the differentiation of (27) with respect to time

$$
\frac{\partial \rho_{1}}{\partial t}=\frac{\partial}{\partial t} \hat{I}[2, \ldots, N] \rho_{N}=\hat{I}[2, \ldots, N] \frac{\partial \rho_{N}}{\partial t} .
$$

Using (24) and (28), we get

$$
\frac{\partial \rho_{1}}{\partial t}=-\hat{I}[2, \ldots, N] \sum_{k=1}^{N}\left(\mathbf{D}_{\mathbf{q}_{\mathbf{k}}}^{\alpha}\left(\mathbf{V}_{k} \rho_{N}\right)+\mathbf{D}_{\mathbf{p}_{\mathbf{k}}}^{\alpha}\left(\mathbf{F}_{k} \rho_{N}\right)\right) .
$$


Let us consider the integration $\hat{I}\left[\mathbf{q}_{k}\right]$ over $\mathbf{q}_{k}$ for $k$ th particle term of Eq. (29),

$$
\begin{gathered}
\hat{I}\left[\mathbf{q}_{k}\right] \mathbf{D}_{\mathbf{q}_{\mathbf{k}}}^{\alpha}\left(\mathbf{V}_{k} \rho_{N}\right)=\hat{I}\left[\mathbf{q}_{k}\right]\left(D_{\mathbf{q}_{k}}^{\alpha} \mathbf{q}_{k}\right)^{-1} D_{\mathbf{q}_{\mathbf{k}}}^{\alpha}\left(\mathbf{V}_{k} \rho_{N}\right)=\Gamma(2-\alpha) \hat{I}\left[\mathbf{q}_{k}\right] \mathbf{q}_{k}^{\alpha-1} D_{\mathbf{q}_{\mathbf{k}}}^{\alpha}\left(\mathbf{V}_{k} \rho_{N}\right)= \\
=\Gamma(\alpha) \Gamma(2-\alpha) \hat{I}^{\alpha}\left[\mathbf{q}_{k}\right] D_{\mathbf{q}_{\mathbf{k}}}^{\alpha}\left(\mathbf{V}_{k} \rho_{N}\right)=\Gamma(\alpha) \Gamma(2-\alpha)\left(\mathbf{V}_{k} \rho_{N}\right)_{-\infty}^{+\infty}=0,
\end{gathered}
$$

where $\hat{I}^{\alpha}\left[\mathbf{q}_{k}\right]$ is a fractional integration with respect to variables $\mathbf{q}_{k}$. In Eq. (30), we use that the distribution $\rho_{N}$ in the limit $\mathbf{q}_{k} \rightarrow \pm \infty$ is equal to zero. It follows from the normalization condition. If the limit is not equal to zero, then the integration over phase space is equal to infinity. Similarly, we obtain

$$
\hat{I}\left[\mathbf{p}_{k}\right] \mathbf{D}_{\mathbf{p}_{\mathbf{k}}}^{\alpha}\left(\mathbf{F}_{k} \rho_{N}\right) \sim\left(\mathbf{F}_{k} \rho_{N}\right)_{-\infty}^{+\infty}=0 .
$$

Then all terms in Eq. (29) with $k=2, \ldots, N$ are equal to zero. Therefore, Eq. (29) are

$$
\frac{\partial \rho_{1}}{\partial t}=-\hat{I}[2, \ldots, N]\left(\mathbf{D}_{\mathbf{q}_{1}}^{\alpha}\left(\mathbf{V}_{1} \rho_{N}\right)+\mathbf{D}_{\mathbf{p}_{\mathbf{1}}}^{\alpha}\left(\mathbf{F}_{1} \rho_{N}\right)\right) \text {. }
$$

The first term in Eq. (31) can be written as

$$
\hat{I}[2, \ldots, N] \mathbf{D}_{\mathbf{q}_{\mathbf{k}}}^{\alpha}\left(\mathbf{V}_{1} \rho_{N}\right)=\mathbf{D}_{\mathbf{q}_{1}}^{\alpha} \mathbf{V}_{1} \hat{I}[2, \ldots, N] \rho_{N}=\mathbf{D}_{\mathbf{q}_{1}}^{\alpha}\left(\mathbf{V}_{1} \rho_{1}\right)
$$

For the binary interactions,

$$
\mathbf{F}_{1}=\mathbf{F}_{1}^{e}+\sum_{k=2}^{N} \mathbf{F}_{1 k}
$$

where $\mathbf{F}_{1}^{e}=\mathbf{F}^{e}\left(\mathbf{q}_{1}, \mathbf{p}_{1}, t\right)$ is the external force, and $\mathbf{F}_{1 k}=\mathbf{F}\left(\mathbf{q}_{1}, \mathbf{p}_{1}, \mathbf{q}_{k}, \mathbf{p}_{k}, t\right)$ are the internal forces. Using (32), the second term in (31) is

$$
\begin{gathered}
\hat{I}[2, \ldots, N] \mathbf{D}_{\mathbf{p}_{\mathbf{1}}}^{\alpha}\left(\mathbf{F}_{1} \rho_{N}\right)=\hat{I}[2, \ldots, N]\left(\mathbf{D}_{\mathbf{p}_{\mathbf{1}}}^{\alpha}\left(\mathbf{F}_{1}^{e} \rho_{N}\right)+\sum_{k=2}^{N} \mathbf{D}_{\mathbf{p}_{\mathbf{1}}}^{\alpha}\left(\mathbf{F}_{1 k} \rho_{N}\right)\right)= \\
=\mathbf{D}_{\mathbf{p}_{\mathbf{1}}}^{\alpha}\left(\mathbf{F}_{1}^{e} \rho_{1}\right)+\sum_{k=2}^{N} \mathbf{D}_{\mathbf{p}_{\mathbf{1}}}^{\alpha} \hat{I}[2, \ldots, N]\left(\mathbf{F}_{1 k} \rho_{N}\right) .
\end{gathered}
$$

We assume that the distribution function is invariant under the permutations of identical particles. Then $\rho_{N}$ is a symmetric function, and all $(N-1)$ terms in Eq. (33) are identical:

$$
\sum_{k=2}^{N} \hat{I}[2, \ldots, N] \mathbf{D}_{\mathbf{p}_{1 \mathbf{s}}}^{\alpha}\left(\mathbf{F}_{1 k} \rho_{N}\right)=(N-1) \hat{I}[2, \ldots, N] \mathbf{D}_{\mathbf{p}_{\mathbf{1}}}^{\alpha}\left(\mathbf{F}_{12} \rho_{N}\right) .
$$

Using $\hat{I}[2, \ldots, N]=\hat{I}[2] \hat{I}[3, \ldots, N]$, we have

$$
\hat{I}[2, \ldots, N] \mathbf{D}_{\mathbf{p}_{\mathbf{1}}}^{\alpha}\left(\mathbf{F}_{12} \rho_{N}\right)=\hat{I}[2] \mathbf{D}_{\mathbf{p}_{\mathbf{1}}}^{\alpha}\left(\mathbf{F}_{12} \hat{I}[3, \ldots, N] \rho_{N}\right)=\mathbf{D}_{\mathbf{p}_{\mathbf{1}}}^{\alpha} \hat{I}[2] \mathbf{F}_{12} \rho_{2},
$$


where

$$
\rho_{2}=\rho\left(\mathbf{q}_{1}, \mathbf{p}_{1}, \mathbf{q}_{2}, \mathbf{p}_{2}, t\right)=\hat{I}[3, \ldots, N] \rho_{N}(\mathbf{q}, \mathbf{p}, t)
$$

is a two-particle distribution function.

Finally, we obtain

$$
\frac{\partial \rho_{1}}{\partial t}+\mathbf{D}_{\mathbf{q}_{1}}^{\alpha}\left(\mathbf{V}_{1} \rho_{1}\right)+\mathbf{D}_{\mathbf{p}_{1}}^{\alpha}\left(\mathbf{F}_{1}^{e} \rho_{1}\right)=I\left(\rho_{2}\right)
$$

where

$$
I\left(\rho_{2}\right)=-(N-1) \mathbf{D}_{\mathbf{p}_{\mathbf{1}}}^{\alpha} \hat{I}[2] \mathbf{F}_{12} \rho_{2}
$$

describes a velocity of particle number change in $4 m$-dimensional two-particle elementary phase volume. This change is caused by the interactions between particles. Equation (37) is the fractional generalization of the first Bogoliubov equation. If $\alpha=1$, then we have the first Bogoliubov equation for non-Hamiltonian systems [39].

\section{Vlasov equation with fractional derivatives}

Let us consider the particles as statistical independent systems. Then

$$
\rho_{2}\left(\mathbf{q}_{1}, \mathbf{p}_{1}, \mathbf{q}_{2}, \mathbf{p}_{2}, t\right)=\rho_{1}\left(\mathbf{q}_{1}, \mathbf{p}_{1}, t\right) \rho_{1}\left(\mathbf{q}_{2}, \mathbf{p}_{2}, t\right)
$$

Substitution of (39) into (38) gives

$$
I\left(\rho_{2}\right)=-\mathbf{D}_{\mathbf{p}_{\mathbf{1}}}^{\alpha} \rho_{1} \hat{I}[2] \mathbf{F}_{12} \rho_{1}\left(\mathbf{q}_{2}, \mathbf{p}_{2}, t\right),
$$

where $\rho_{1}=\rho_{1}\left(\mathbf{q}_{1}, \mathbf{p}_{1}, t\right)$.

Let us define

$$
\mathbf{F}^{e f f}\left(\mathbf{q}_{1}, \mathbf{p}_{1}, t\right)=\hat{I}[2] \mathbf{F}_{12} \rho_{1}\left(\mathbf{q}_{2}, \mathbf{p}_{2}, t\right)
$$

Then,

$$
I\left(\rho_{2}\right)=-\mathbf{D}_{\mathbf{p}_{1}}^{\alpha}\left(\rho_{1} \mathbf{F}^{e f f}\right) .
$$

Substituting of (41) into (37), we obtain

$$
\frac{\partial \rho_{1}}{\partial t}+\mathbf{D}_{\mathbf{q}_{1}}^{\alpha}\left(\mathbf{V}_{1} \rho_{1}\right)+\mathbf{D}_{\mathbf{p}_{1}}^{\alpha}\left(\left(\mathbf{F}_{1}^{e}+(N-1) \mathbf{F}^{e f f}\right) \rho_{1}\right)=0
$$

that is a closed equation for the one-particle distribution function with the external force $\mathbf{F}_{1}^{e}$ and the effective force $\mathbf{F}^{e f f}$. Equation (42) is the fractional generalization of the Vlasov equation [34, 35] that has phase-space derivatives of noninteger order. For $\alpha=1$, we get the Vlasov equation for the non-Hamiltonian systems [39]. 


\section{Fokker-Planck-Zaslavsky equation for phase-space}

The Fokker-Planck equations with fractional coordinate derivatives have been suggested by Zaslavsky [12] to describe chaotic dynamics. It is known that Fokker-Planck equation can be derived from the Liouville equation [40, 41, 42]. In this section, we obtain Fokker-PlanckZaslavsky equation that has fractional derivatives in phase space.

Let us consider a system of $N$ identical particles and the Brownian particle that is described by the distribution function

$$
\rho_{N+1}=\rho_{N+1}(\mathbf{q}, \mathbf{p}, Q, P, t)
$$

where

$$
\begin{array}{ll}
\mathbf{q}=\left(\mathbf{q}_{1}, \ldots, \mathbf{q}_{N}\right), & \mathbf{q}_{k}=\left(q_{k 1}, \ldots, q_{k m}\right), \\
\mathbf{p}=\left(\mathbf{p}_{1}, \ldots, \mathbf{p}_{N}\right), & \mathbf{p}_{k}=\left(p_{k 1}, \ldots, p_{k m}\right)
\end{array}
$$

are the coordinates and momenta of the particles; $Q=\left(Q_{s}\right)$ and $P=\left(P_{s}\right)(s=1, \ldots, m)$ are Brownian particle coordinates and momenta. The normalization condition is

$$
\hat{I}[1, \ldots, N, N+1] \rho_{N+1}=1 .
$$

The distribution function for the Brownian particle is defined by

$$
\rho_{B}(Q, P, t)=\hat{I}[1, \ldots, N] \rho_{N+1}(\mathbf{q}, \mathbf{p}, Q, P, t) .
$$

The Liouville equation for $\rho_{N+1}$ is

$$
\frac{\partial \rho_{N+1}}{\partial t}-i\left(L_{N}+L_{B}\right) \rho_{N+1}=0
$$

where

$$
\begin{aligned}
-i L_{N} \rho & =\sum_{k, s}^{N, m}\left(\mathbf{D}_{q_{k s}}^{\alpha}\left(G_{s}^{k} \rho\right)+\mathbf{D}_{p_{k s}}^{\alpha}\left(F_{s}^{k} \rho\right)\right), \\
-i L_{B} \rho & =\sum_{k, s}^{N, m}\left(\mathbf{D}_{Q_{s}}^{\alpha}\left(g_{s} \rho\right)+\mathbf{D}_{P_{s}}^{\alpha}\left(f_{s} \rho\right)\right) .
\end{aligned}
$$

Here, $L_{N}$ and $L_{B}$ are Liouville operators with fractional derivatives, and

$$
\mathbf{D}_{A}^{\alpha} B=\left(D_{A}^{\alpha} A\right)^{-1} D_{A}^{\alpha} B .
$$

The functions $G_{s}^{k}$ and $F_{s}^{k}$ are defined by the equations of motion for particle,

$$
\frac{d q_{k s}}{d t}=G_{s}^{k}(\mathbf{q}, \mathbf{p}), \quad \frac{d p_{k s}}{d t}=F_{s}^{k}(\mathbf{q}, \mathbf{p}, Q, P), \quad k=1, \ldots, N
$$


The Hamilton equations for the Brownian particle

$$
\frac{d Q_{s}}{d t}=g_{s}(Q, P), \quad \frac{d P_{s}}{d t}=f_{s}(\mathbf{q}, \mathbf{p}, Q, P)
$$

define $g_{s}$ and $f_{s}$.

Let us use the boundary condition in the form

$$
\lim _{t \rightarrow-\infty} \rho_{N+1}(\mathbf{q}, \mathbf{p}, Q, P, t)=\rho_{N}(\mathbf{q}, \mathbf{p}, Q, T) \rho_{B}(Q, P, t)
$$

where

$$
\rho_{N}(\mathbf{q}, \mathbf{p}, Q, T)=\exp \beta(\mathcal{F}-H(\mathbf{q}, \mathbf{p}, Q))
$$

is the canonical Gibbs distribution for

$$
H(\mathbf{q}, \mathbf{p}, Q)=H_{N}(\mathbf{q}, \mathbf{p})+\sum_{k=1}^{N} U_{B}\left(\mathbf{q}_{k}, Q\right)
$$

Here, $H_{N}$ is a Hamiltonian of an $n$-particle system, and $U_{B}$ is an energy of interaction between particles and Brownian particle. If we suppose

$$
G_{s}^{k}=p_{k s} / m, \quad g_{s}=P_{s} / M
$$

then

$$
H_{N}(\mathbf{q}, \mathbf{p})=\sum_{k, s}^{N, m} \frac{p^{2}}{2 m}+\sum_{k<l} U\left(\mathbf{q}_{k}, \mathbf{q}_{l}\right) .
$$

The boundary condition (50) can be realized [36] by the infinitesimal source term in the Liouville equation:

$$
\frac{\partial \rho_{N+1}}{\partial t}-i\left(L_{N}+L_{B}\right) \rho_{N+1}=-\varepsilon\left(\rho_{N+1}-\rho_{N} \rho_{B}\right)
$$

Integrating (55) by $\hat{I}[1, \ldots, N]$, we obtain

$$
\frac{\partial \rho_{B}}{\partial t}+\sum_{s=1}^{m} \mathbf{D}_{Q_{s}}^{\alpha}\left(g_{s} \rho_{B}\right)+\hat{I}[1, \ldots, N] \sum_{s=1}^{m} \mathbf{D}_{P_{s}}^{\alpha}\left(f_{s} \rho_{N+1}\right)=0,
$$

which is the Liouville equation for reduced distribution function of the Brownian particle.

The formal solution of Eq. (55) has the form

$$
\rho_{N+1}(t)=\rho_{B}(t) \rho_{N}-\int_{-\infty}^{0} d \tau e^{\varepsilon \tau} e^{-i \tau\left(L_{N}+L_{B}\right)}\left(\frac{\partial}{\partial \tau}-i\left(L_{N}+L_{B}\right)\right) \rho_{B}(t+\tau) \rho_{N}
$$


Substituting (57) into (156), we get

$$
\begin{gathered}
\frac{\partial \rho_{B}}{\partial t}+\sum_{s=1}^{m} \mathbf{D}_{Q_{s}}^{\alpha}\left(g_{s} \rho_{B}\right)+\sum_{s=1}^{m} \mathbf{D}_{P_{s}}^{\alpha} \rho_{B} \hat{I}[1, \ldots, N]\left(f_{s} \rho_{N}\right)- \\
-\hat{I}^{\alpha}[1, \ldots, N] \sum_{s=1}^{m} \mathbf{D}_{P_{s}}^{\alpha} \int_{-\infty}^{0} d \tau e^{\varepsilon \tau} e^{-i \tau\left(L_{N}+L_{B}\right)}\left(\frac{\partial}{\partial \tau}-i\left(L_{N}+L_{B}\right)\right) \rho_{B}(t+\tau) \rho_{N}=0 .
\end{gathered}
$$

The expression $\hat{I}[1, . ., N] f_{s} \rho_{N}$ can be considered as average value of the force $f_{s}$. For the canonical Gibbs distribution (51) it is equal to zero. Using

$$
\mathbf{D}_{Q_{s}}^{\alpha} \rho_{N}=\frac{1}{k T} f_{s}^{(p)} \rho_{N}
$$

where $f_{s}^{(p)}$ is a fractional potential force [21]:

$$
f_{s}^{(p)}=-\mathbf{D}_{Q_{s}}^{\alpha} U_{B}
$$

we have

$$
-i L_{B} \rho_{N+1}=\left(\frac{P_{s} f_{s}^{(p)}}{M k T} \rho_{B}+\mathbf{D}_{Q_{s}}^{\alpha}\left(g_{s} \rho_{B}\right)+\mathbf{D}_{P_{s}}^{\alpha}\left(f_{s} \rho_{B}\right)\right) \rho_{N} .
$$

It can be proved by integration that the term

$$
\frac{\partial \rho_{B}}{\partial t}+\mathbf{D}_{Q_{s}}^{\alpha}\left(g_{s} \rho_{B}\right)
$$

in Eq. (58) does not contribute. Then (158) gives

$$
\begin{gathered}
\frac{\partial \rho_{B}}{\partial t}+\sum_{s=1}^{m} \mathbf{D}_{Q_{s}}^{\alpha}\left(g_{s} \rho_{B}\right)+\sum_{s=1}^{m} \mathbf{D}_{P_{s}}^{\alpha} \hat{I}[1, \ldots, N] \int_{-\infty}^{0} d \tau e^{\varepsilon \tau} f_{s} e^{-i \tau\left(L_{N}+L_{B}\right)} \rho_{N} \cdot \\
\cdot\left(\mathbf{D}_{P_{s^{\prime}}}^{\alpha}\left(f_{s^{\prime}} \rho_{B}(t+\tau)\right)+\frac{\beta f_{s^{\prime}} P_{s^{\prime}}}{M} \rho_{B}(t+\tau)\right)=0 .
\end{gathered}
$$

Equation (62) is a closed integro-differential equation for the distribution function $\rho_{B}$. Note that $f_{s}$ can be presented as

$$
f_{s}=f_{s}^{(p)}+f_{s}^{(n)}
$$

where $f_{s}^{(p)}$ is a potential force (마) , and $f_{s}^{(n)}$ is a non-potential force that acts on the Brownian particle. For the equilibrium approximation $P \sim(M k T)^{1 / 2}, i L_{B} \sim M^{-1 / 2}$ and $i L_{N} \sim m^{-1 / 2}$. If $M>>m$, we can use perturbation theory.

Using the approximation $\rho_{B}(t+\tau)=\rho_{B}(t)$ for Eq. (62), we obtain

$$
\frac{\partial \rho_{B}}{\partial t}+\sum_{s=1}^{m} \mathbf{D}_{Q_{s}}^{\alpha}\left(g_{s} \rho_{B}\right)+\sum_{s=1}^{m} \mathbf{D}_{P_{s}}^{\alpha}\left(\frac{M}{\beta} \mathbf{D}_{P_{s^{\prime}}}^{\alpha}\left(\gamma_{s s^{\prime}}^{1} \rho_{B}(t)\right)+\gamma_{s s^{\prime}}^{2} P_{s^{\prime}} \rho_{B}(t)\right)=0
$$


where

$$
\begin{aligned}
& \gamma_{s s^{\prime}}^{1}=\beta M \hat{I}^{\alpha}[1, \ldots, N] \int_{-\infty}^{0} d \tau e^{\varepsilon \tau} f_{s} e^{-i \tau L_{N}} f_{s^{\prime}} \rho_{N}, \\
& \gamma_{s s^{\prime}}^{2}=\beta M \hat{I}^{\alpha}[1, \ldots, N] \int_{-\infty}^{0} d \tau e^{\varepsilon \tau} f_{s} e^{-i \tau L_{N}} f_{s^{\prime}}^{(p)} \rho_{N} .
\end{aligned}
$$

If $f_{s}=f_{s}^{(p)}$, then $\gamma_{s s^{\prime}}^{1}=\gamma_{s s^{\prime}}^{2}$. As a result, we derive the Fokker-Planck-Zaslavsky equation [12, 9] for the phase space.

\section{Linear fractional kinetic equation}

Let us consider Eq. (37) with $I\left(\rho_{2}\right)=0, \mathbf{V}_{1}=\mathbf{p} / m=\mathbf{v}$, and $\mathbf{F}^{e}=e \mathbf{E}, \mathbf{B}=0$. Then

$$
\frac{\partial \rho_{1}}{\partial t}+\left(\mathbf{v}, \mathbf{D}_{\mathbf{q}}^{\alpha} \rho_{1}\right)+e\left(\mathbf{E}, \mathbf{D}_{\mathbf{p}}^{\alpha} \rho_{1}\right)=0,
$$

where

$$
\left(\mathbf{v}, \mathbf{D}_{\mathbf{q}}^{\alpha} \rho\right)=\sum_{s=1}^{m}\left(v_{s}, \mathbf{D}_{\mathbf{q}_{s}}^{\alpha} \rho\right) .
$$

If we take into account the magnetic field $(\mathbf{B} \neq 0)$, then we must use the fractional generalization of Leibnitz rules

$$
\mathbf{D}_{\mathbf{p}}^{\alpha}(f g)=\sum_{s=0}^{\infty} \frac{\Gamma(\alpha+1)}{\Gamma(s+1) \Gamma(\alpha-s+1)}\left(\mathbf{D}_{\mathbf{p}}^{\alpha-s} f\right) \mathbf{D}_{\mathbf{p}}^{s} g,
$$

where $s$ are integer numbers. In this case, Eq. (66) has the addition term

$$
\begin{gathered}
\frac{e}{m c} \mathbf{D}_{\mathbf{p}}^{\alpha}\left([\mathbf{p}, \mathbf{B}] \rho_{1}\right)=\frac{e}{m c} \sum_{k l m} \mathbf{D}_{p_{k}}^{\alpha}\left(\varepsilon_{k l m} p_{l} B_{m} \rho_{1}\right)=\frac{e}{m c} \sum_{k l m} \varepsilon_{k l m} B_{m} \mathbf{D}_{p_{k}}^{\alpha}\left(p_{l} \rho_{1}\right)= \\
=\frac{e}{m c} \sum_{k l m} \varepsilon_{k l m} B_{m} \sum_{i=0}^{1} \frac{\Gamma(\alpha+1)}{\Gamma(i+1) \Gamma(\alpha-i+1)}\left[\mathbf{D}_{p_{k}}^{\alpha-i} \rho_{1}\right] \delta_{k l} p_{l}^{i}= \\
=\frac{e}{m c} \sum_{k l m} \varepsilon_{k l m} B_{m}\left(\left[\mathbf{D}_{p_{k}}^{\alpha} \rho_{1}\right] p_{l}+\alpha\left[\mathbf{D}_{p_{k}}^{\alpha-1} \rho\right] \delta_{k l}\right)= \\
\frac{e}{m c} \sum_{k l m} \varepsilon_{k l m} B_{m} p_{l}\left[\mathbf{D}_{p_{k}}^{\alpha} \rho_{1}\right]=\frac{e}{m c}\left(\left(\mathbf{D}_{p_{k}}^{\alpha} \rho_{1}\right),[\mathbf{p}, \mathbf{B}]\right) .
\end{gathered}
$$

Let us consider the perturbation [37, 38] of the distribution function in the form

$$
\rho_{1}=\tilde{\rho}_{1}+\delta \rho_{1}(t, q, p),
$$


where $\tilde{\rho}_{1}$ is a homogeneous stationary density of probability that satisfies Eq. (66) for $\mathbf{E}=0$. Substituting (70) into Eq. (6ㅏ), we get

$$
\frac{\partial \delta \rho_{1}}{\partial t}+\left(\mathbf{v}, \mathbf{D}_{\mathbf{q}}^{\alpha} \delta \rho_{1}\right)+e\left(\mathbf{E}, \mathbf{D}_{\mathbf{p}}^{\alpha} \tilde{\rho}_{1}\right)=0
$$

Equation (171) is linear fractional kinetic equation for the first perturbation $\delta \rho_{1}$ of the distribution function. Solutions of fractional linear kinetic equations (71) are considered in Ref. [13]. For $\mathbf{E}=0$, the function $\delta \rho_{1}$ is described by the function

$$
\left(g_{s} t\right)^{-1 / \alpha} L_{\alpha}\left[q_{s}\left(g_{s} t\right)^{-1 / \alpha}\right]
$$

where $g_{s}=v_{s}\left(D_{q_{s}}^{\alpha} q_{s}\right)^{-1}$, and

$$
L_{\alpha}[x]=\frac{1}{2 \pi} \int_{-\infty}^{+\infty} d k e^{-i k x} e^{-a|k|^{\alpha}}
$$

is the Levy stable p.d.f. [43]. The examples of $L_{\alpha}[x]$ are shown in Fig. 1.

For $\alpha=1$, the function (73) gives the Cauchy distribution

$$
L_{1}[x]=\frac{1}{\pi} \frac{1}{x^{2}+1}
$$

and (172) is

$$
\frac{1}{\pi} \frac{\left(g_{s} t\right)^{-1}}{q_{s}^{2}\left(g_{s} t\right)^{-2}+1}
$$

For $\alpha=2$, Eq. (73) gives the Gauss distribution:

$$
L_{2}[x]=\frac{1}{2 \sqrt{\pi}} e^{-x^{2} / 4},
$$

and the function (72) is

$$
\left(g_{s} t\right)^{-1 / 2} \frac{1}{2 \sqrt{\pi}} e^{-q_{s}^{2} /\left(4 g_{s} t\right)} .
$$

For $1<\alpha \leq 2$, the function $L_{\alpha}[x]$ can be presented as the expansion

$$
L_{\alpha}[x]=-\frac{1}{\pi x} \sum_{n=1}^{\infty}(-x)^{n} \frac{\Gamma(1+n / \alpha)}{n !} \sin (n \pi / 2) .
$$

The asymptotic $(x \rightarrow \infty, 1<\alpha<2)$ is given by

$$
L_{\alpha}[x] \sim-\frac{1}{\pi x} \sum_{n=1}^{\infty}(-1)^{n} x^{-n \alpha} \frac{\Gamma(1+n \alpha)}{n !} \sin (n \pi / 2) .
$$

As the result, the asymptotic of the solution, exhibits the power-like tails for $x \rightarrow \infty$. This tails is the important property of solutions of equations with the noninteger derivative. 


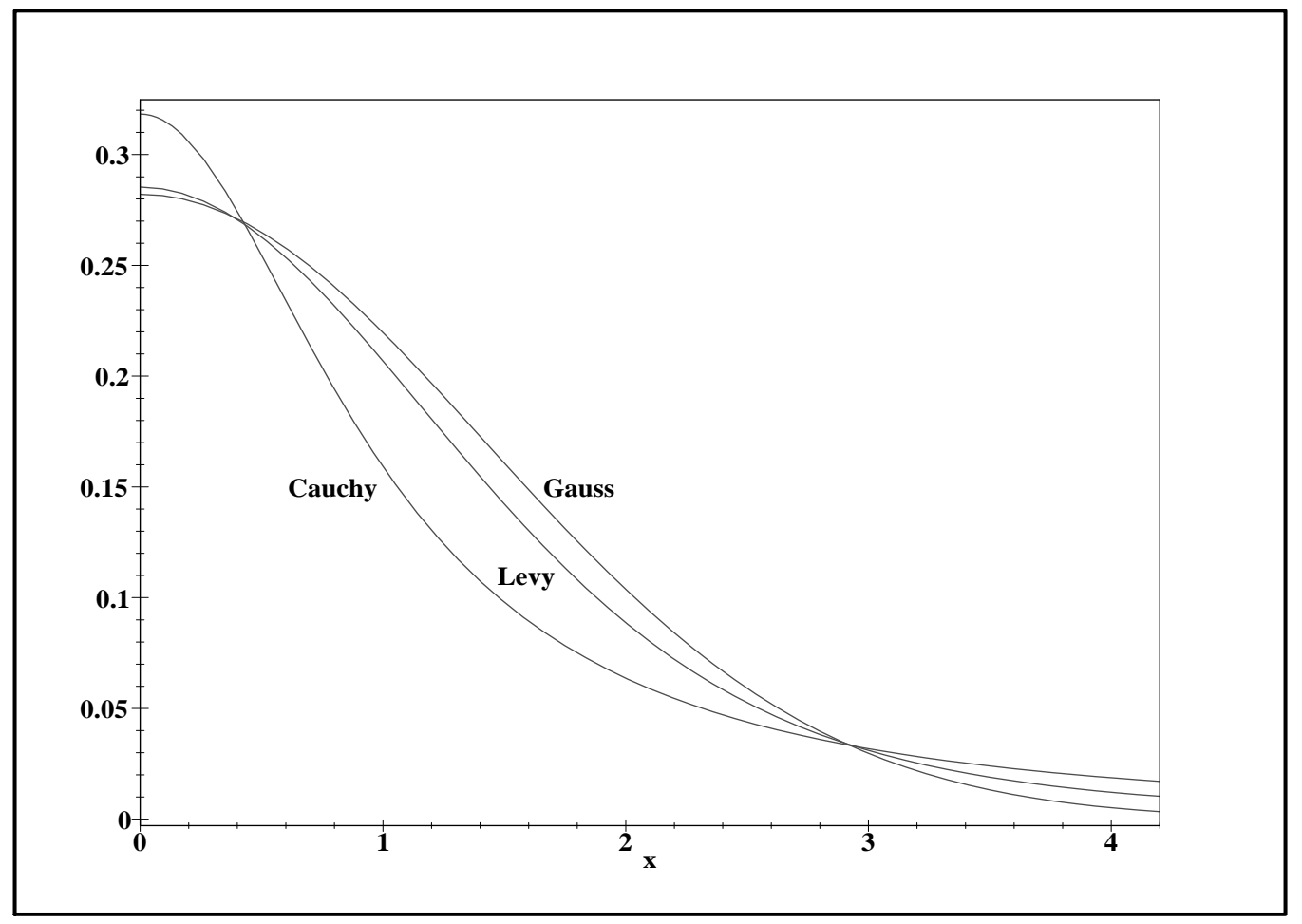

Figure 1: Gauss p.d.f. $(\alpha=2)$, Levy p.d.f. $(\alpha=1.6)$, and Cauchy p.d.f. $(\alpha=1.0)$. Levy for $\alpha=1.6$ lies between Cauchy and Gauss p.d.f. In the asymptotic $x \rightarrow \infty$ and $x>3$ on the plot, the upper curve is Cauchy p.d.f., and the lower curve is the Gauss p.d.f. 


\section{Conclusion}

In this paper, we consider equations with derivatives of noninteger order that can be used in statistical mechanics and physical kinetics. We derive the Liouville, Bogoliubov, Vlasov and Fokker-Planck equations with fractional derivatives with respect to coordinates and momenta. To derive the fractional Liouville equation (FLE), we consider the conservation of probability in the fractional differential volume element. This element can be considered as a small part of the phase-space set with noninteger dimension. Using the FLE, we obtain a fractional generalization of the Bogoliubov hierarchy equations. These equations describe the evolution of the reduced density of probability in the fractional phase-space volume element. Fractional Bogoliubov equations can be used to derive fractional kinetic equations [5, 12, 13]. In this paper, we obtain Fokker-Planck-Zaslavsky equation, fractional Vlasov and linear kinetic equations.

The fractional kinetics is related to the equations that contains derivatives of noninteger order. These equations appear in the description of chaotic dynamics, and the fractal media. The fractional derivatives can be connected with long-range power-law interaction of the systems [23, 24, 25]. For noninteger derivatives with respect to coordinates, we have the power-like tails as the important property of the solutions of the fractional equations.

\section{References}

[1] I. Podlubny, Fractional Differential Equations (Academic Press, New York, 1999)

[2] A.A. Kilbas, H.M. Srivastava, J.J. Trujillo, Theory and Applications of Fractional Differential Equations (Elsevier, New York, 2006)

[3] S.G. Samko, A.A. Kilbas, O.I. Marichev, Fractional Integrals and Derivatives Theory and Applications (Gordon and Breach, New York, 1993)

[4] K.B. Oldham, J. Spanier, The Fractional Calculus (Academic Press, New York, 1974)

[5] G.M. Zaslavsky, "Chaos, fractional kinetics, and anomalous transport" Phys. Rep. 371, 461-580 (2002).

[6] G.M. Zaslavsky, Hamiltonian Chaos and Fractional Dynamics (Oxford University Press, Oxford, 2005)

[7] A. Carpinteri, F. Mainardi, Fractals and Fractional Calculus in Continuum Mechanics (Springer, New York, 1997)

[8] V.E. Tarasov, "Continuous medium model for fractal media" Phys. Lett. A 336, 167174 (2005); "Fractional hydrodynamic equations for fractal media" Ann. Phys. 318, 286-307 (2005); "Possible experimental test of continuous medium model for fractal media" Phys. Lett. A 341, 467-472 (2005). 
[9] V.E. Tarasov, "Fractional Fokker-Planck equation for fractal media" Chaos 15, 023102 (2005).

[10] N. Laskin, "Fractals and quantum mechanics" Chaos 10, 780-790 (2000); "Fractional quantum mechanics" Phys. Rev. E 62, 3135-3145 (2000); "Fractional quantum mechanics and Levy path integrals" Phys. Lett. A 268, 298-305 (2000); "Fractional Schrodinger equation" Phys. Rev. E 66, 056108 (2002).

[11] M. Naber, "Time fractional Schrodinger equation" J. Math. Phys. 45, 3339-3352 (2004).

[12] G.M. Zaslavsky, "Fractional kinetic equation for Hamiltonian chaos" Physica D 76, 110-122 (1994).

[13] A.I. Saichev, G.M. Zaslavsky, "Fractional kinetic equations: solutions and applications" Chaos 7, 753-764 (1997).

[14] G.M. Zaslavsky, M.A. Edelman, "Fractional kinetics: from pseudochaotic dynamics to Maxwell's demon" Physica D 193, 128-147 (2004).

[15] R.R. Nigmatullin, "Fractional kinetic equations and universal decoupling of a memory function in mesoscale region" Physica A 363, 282-298 (2006); A.V. Chechkin, V.Yu. Gonchar, M. Szydlowsky, "Fractional kinetics for relaxation and superdiffusion in magnetic field" Physics of Plasmas 9, 78-88 (2002); R.K. Saxena, A.M. Mathai, H.J. Haubold, "On fractional kinetic equations" Astrophysics and Space Science, 282, 281-287 (2002).

[16] B.A. Carreras, V.E. Lynch, G.M. Zaslavsky, "Anomalous diffusion and exit time distribution of particle tracers in plasma turbulence model" Physics of Plasmas 8, 5096-5103 (2001).

[17] V.E. Tarasov, "Electromagnetic field of fractal distribution of charged particles" Physics of Plasmas 12, 082106 (2005); "Multipole moments of fractal distribution of charges" Mod. Phys. Lett. B 19, 1107-1118 (2005); "Magnetohydrodynamics of fractal media" Physics of Plasmas 13, 052107 (2006).

[18] F. Mainardi, R. Gorenflo, "On Mittag-Leffler-type functions in fractional evolution processes" J. Comput. Appl. Math. 118, 283-299 (2000).

[19] F. Mainardi, "Fractional relaxation-oscillation and fractional diffusion-wave phenomena" Chaos, Solitons and Fractals 7, 1461-1477 (1996).

[20] V.E. Tarasov, G.M. Zaslavsky, "Dynamics with low-level fractionality" Physica A 368 (2006) 399-415.

[21] V.E. Tarasov, "Fractional generalization of Liouville equation" Chaos 14, 123-127 (2004); "Fractional systems and fractional Bogoliubov hierarchy equations" Phys. Rev. E 71, 011102 (2005); "Fractional Liouville and BBGKI equations" J. Phys. Conf. Ser. 
7, 17-33 (2005); "Transport equations from Liouville equations for fractional systems" Int. J. Mod. Phys. B 20, 341-354 (2006).

[22] V.E. Tarasov, "Fractional generalization of gradient and Hamiltonian systems" J. Phys. A 38, 5929-5943 (2005); "Fractional generalization of gradient systems" Lett. Math. Phys. 73, 49-58 (2005); "Fractional variations for dynamical systems: Hamilton and Lagrange approaches" J. Phys. A 39, 8409-8425 (2006).

[23] N. Laskin, G.M. Zaslavsky, "Nonlinear fractional dynamics on a lattice with long-range interactions" Physica A 368, 38-54 (2006).

[24] V.E. Tarasov, G.M. Zaslavsky, "Fractional dynamics of coupled oscillators with longrange interaction" Chaos 16, 023110 (2006). "Fractional dynamics of systems with longrange interaction" Commun. Nonlin. Sci. Numer. Simul. 11, 885-898 (2006).

[25] N. Korabel, G.M. Zaslavsky, V.E. Tarasov, "Coupled oscillators with power-law interaction and their fractional dynamics analogues" Commun. Nonlin. Sci. Numer. Simul. 12, 1405-1417 (2007); math-ph/0603074).

[26] E.W. Montroll, M.F. Shlesinger, "The wonderful world of random walks" In: Studies in Statistical Mechanics, Vol. 11. J. Lebowitz, E. Montroll (Eds.), (North-Holland, Amsterdam, 1984) pp.1-121.

[27] V.V. Uchaikin, "Self-similar anomalous diffusion and Levy-stable laws" Physics-Uspekhi 46, 821-849 (2003); "Anomalous diffusion and fractional stable distributions" J. Exper. Theor. Phys. 97, 810-825 (2003).

[28] K. Cottrill-Shepherd, M. Naber, "Fractional differential forms" J. Math. Phys. 42, 2203-2212 (2001).

[29] M. Caputo, "Linear models of dissipation whose Q is almost frequency independent" Geophys. J. Royal Astron. Soc. 13, 529-539 (1967).

[30] N.N. Bogoliubov, "Kinetic equations" Zh. Exper. Teor. Fiz. 16, 691-702 (1946); J. Phys. USSR 10, 265 (1946).

[31] K.P. Gurov, Foundation of Kinetic Theory. Method of N.N. Bogoliubov (Nauka, Moscow, 1966) in Russian

[32] D.Ya. Petrina, V.I. Gerasimenko, P.V. Malishev, Mathematical Foundation of Classical Statistical Mechanics (Naukova dumka, Kiev, 1985) in Russian.

[33] G.A. Martynov, Classical Statistical Mechanics (Kluwer, Dordrecht, 1997)

[34] A.A. Vlasov, "Vibrating properties of electronic gas" Zh. Exper. Teor. Fiz. 8, 291 (1938); "On the kinetic theory of an assembly of particles with collective interaction" J. Phys. USSR 9, 25 (1945).

[35] A.A. Vlasov, Many-particle Theory and its Application to Plasma (Gordon and Breach, New York, 1961) 
[36] D.N. Zubarev, M.Yu. Novikov, "Generalized formulation of the boundary condition for the Liouville equation and for BBGKY hierarchy" Teor. Mat. Fiz. 13, 406-420 (1972).

[37] G. Ecker, Theory of Fully Ionized Plasmas (Academic Press, New York, 1972)

[38] N.A. Krall, A.W. Trivelpiece, Principles of Plasma Physics (McGraw-Hill, New York, 1973)

[39] V.E. Tarasov, "Stationary solution of Liouville equation for non-Hamiltonian systems" Ann. Phys. 316, 393-413 (2005); "Classical canonical distribution for dissipative systems" Mod. Phys. Lett. B 17, 1219-1226 (2003).

[40] A. Isihara, Statistical Physics (Academic Press, New York, 1971) App. IV. Sec. 7.5.

[41] P. Resibois, M. De Leener, Classical Kinetic Theory of Fluids (Wyley, New York, 1977) Sec. IX.4.

[42] D. Forster, Hydrodynamics Fluctuations, Broken Symmetry, and Correlation Functions (Benjamin, London, 1975) Sec. 6.4.

[43] V. Feller, An Introduction to Probability Theory and its Applications (Wiley, New York, 1971) Vol. 2. 